\title{
Children infected with meningitis before 1 year of age were at increased risk for disability at 5 years of age
}

Bedford H, de Louvois J, Halket S, et al. Meningitis in infancy in England and Wales: follow up at age 5 years. BMJ 2001 Sep 8;323:533-6.

\section{QUESTION: In children who survived meningitis during their first year of life, what are the sequelae at 5 years of age?}

Design

Inception cohort of children who survived an acute attack of meningitis in their first year compared with children who did not have meningitis.

\section{Setting}

General practices in England and Wales, UK.

\section{Patients}

1717 children who contracted meningitis during their first year. Sex- and age-matched children from the same general practice as the index child formed the control group. Data at 5 years of age were available for 1584 children (92\%) who had had meningitis and 1391 in the control group (94\%).

\section{Assessment of prognostic factors}

General practitioners completed questionnaires on children that described any problems in neuromotor development, learning, vision, hearing, speech and language, and behaviour, and the presence of seizure disorders. Parents completed questionnaires on their child's health, development, and schooling. Gestational age, birthweight, the organism that caused the meningitis, and age at time of infection were also recorded.

\section{Main outcome measures}

Disability (none to severe) at 5 years of age. Children with no disability had no developmental problems. Mild disability included middle ear disease, strabismus, febrile convulsions, and behavioural problems. Moderate disability included mild neuromotor disabilities, intellectual impairment, moderate sensorineural hearing loss, mild to moderate visual impairment, treatment controlled epilepsy, and uncomplicated hydrocephalus. Severe disability included severe neuromotor and intellectual impairment, severe seizure disorders, and severe visual or auditory impairment.

For correspondence: $M$ $H$ Bedford, Institute of Child Health, London, $U K$.

h.bedford@ich.ucl.ac.uk.

A modified version of this abstract also

appears in

Evidence-Based

Nursing. disorders were 461 and $275 \quad(\mathrm{p}<0.001)$ (table). Children presenting with meningitis within the first month of life had more health and developmental problems than did those infected after 1 month of age $(p=0.03)$. Severity of disability varied according to the type of organism responsible for the infection.

\section{Conclusion}

Children who had meningitis in their first year of life had a 10-fold increase over non-infected children in the risk for moderate or severe disability at 5 years of age.

\section{COMMENTARY}

The study by Bedford et al provides insight into the range of problems experienced by children who had meningitis in infancy and were born between 1985 and 1987 in England and Wales, UK. The authors conclude that meningitis in infancy has serious consequences. The strengths of the study are its sample size (1584 children), its prospective design, the fact that it was national in scope, and the relatively long follow up period of 5 years (although age 5 years is still too early to capture some important learning disabilities). Infants who were $<28$ days old when they had meningitis had worse outcome than those who had meningitis between 29 and 365 days of age.

The broad inclusion criteria of the study represent both a strength and a limitation. Infants with predictable disability (eg, infants with the Down syndrome or anomalies of the central nervous system, excluding those with spina bifida or ventricular shunt infections) were included in the meningitis group but not in the control group, and were thus overrepresented in the former. The inclusion of premature infants of all gestational ages similarly permits generalisation to the population of England and Wales but blurs differences between patient subgroups. The authors cast a wide net with respect to the cause of meningitis and included viral and fungal infections.

This study paints a picture on a broad and representative landscape of the outcomes of infants who have had meningitis. Physicians who are called on to provide families with prognostic information about their children will be more confident about the outcome data described in this study than they were before. Although predictive models that account for the severity of meningitis can increase the accuracy of prognostications, caution is advised with regard to individualising prognostic information on the basis of this study. ${ }^{1}$ Improved prediction of outcomes of uncommon conditions await the development of national registries, which collect detailed information about the causative illness, and linkage to large administrative databases from which long term outcomes can be ascertained.

Max Perlman, MD

Hospital for Sick Children, Toronto, Ontario, Canada

1 Klinger G, Chin CN, Beyene J, Perlman M. Predicting the outcome of neonatal bacterial meningitis. Pediatrics 2000; 106:477-82.

\begin{tabular}{llcc} 
Severity of disability at $\mathbf{5}$ years & Meningitis & Control & Relative risk (95\% CI) \\
Severe or moderate & $16 \%$ & $1.5 \%$ & $10.33(6.60$ to 16.0$)$ \\
\hline Mild & $29 \%$ & $20 \%$ & $1.47(1.29$ to 1.68$)$ \\
\hline None & $55 \%$ & $79 \%$ & $0.70(0.67$ to 0.74$)$ \\
\hline
\end{tabular}

